\title{
Linewidth Reduction and Frequency Stabilization of Semiconductor Lasers Using Dispersive Losses in an Atomic Vapor
}

\author{
John M. Iannelli, Y. Shevy, J. Kitching, and Amnon Yariv, Fellow, IEEE
}

\begin{abstract}
The effects of a dispersive loss on the linewidth and chirp of a semiconductor laser are discussed. A van der PoI analysis is used to obtain expressions for linewidth reduction and chirp reduction in an arbitrary optical feedback configuration. Specifically the use of an atomic cesium vapor as a dispersive loss mechanism is considered. The dominant factor in reducing the linewidth and chirp is shown to be the frequencydependent phase change from the atomic vapor. However, we also show that under certain operating conditions the frequency-dependent amplitude changes from the atomic vapor also contribute to the reduction. The results are shown to be in agreement with a detailed rate equation calculation.
\end{abstract}

\section{INTRODUCTION}

$\mathrm{T}$ HE spectral purity of semiconductor lasers is a topic of great interest. Due to the importance of the semiconductor laser linewidth in many device applications (interferometric fiber sensors, heterodyne detectors, coherent transmission systems) much effort has been devoted to its study and reduction. Fundamentally, the laser linewidth is a direct consequence of the diffusion of the phase of the optical field with time. In semiconductor lasers, one of the primary driving mechanisms of phase diffusion is spontaneous emission into the lasing mode. A limit derived by Schawlow and Townes [1] shows the linewidth to be inversely proportional to both the output power and the square of the optical. mode volume. Due to the small $Q$ cavity of semiconductor lasers and the additional effect of amplitude to phase coupling described by the $\alpha$ parameter [2], linewidths are typically on the order of tens of $\mathrm{MHz}$.

Several methods have been proposed and demonstrated for reducing semiconductor laser linewidths. We have recently shown both theoretically and experimentally that the incorporation of a dispersive loss mechanism in the laser cavity can lead to a dramatic quenching of the spectral linewidth [3], [4]. Due to the strong amplitude to phase coupling in semiconductor lasers, a dispersive loss can be used to reduce the linewidth by producing changes

Manuscript received May 26, 1992; revised October 12, 1992. This work was supported by DARPA, ONR, and AFOSR. The work of J. Kitching was supported by NSERC Canada.

The authors are with the California Institute of Technology, Pasadena, CA 91125 .

IEEE Log Number 9207853. in the field's amplitude and phase which correct for the initial phase fluctuation. The key quantities in this method are the slopes of the loss rate and the accompanying refractive index versus frequency. The correct slope produces negative as opposed to positive feedback to phase fluctuations, the magnitude of the slope determines the strength of the correction signal, and in the case of amplitude changes, the $\alpha$ parameter governs the coupling of the amplitude to the phase. Ironically, the amplitude to phase coupling mechanism which normally increases the linewidth by a factor of $\left(1+\alpha^{2}\right)$ ( $\alpha$ typically in the range of 3-6) [2] can, in conjunction with a dispersive loss, be used as leverage for obtaining linewidth reduction.

In addition to reducing the linewidth, a dispersive loss also improves the frequency stability through a reduction of adiabatic chirp. As will be discussed below, the frequency stability is improved with respect to changes in injection current as well as variations in other external parameters such as cavity length. It will also be shown that linewidth reduction and adiabatic chirp reduction are simply related to each other [5].

As stated above, the field corrections made by the dispersive loss are twofold. Analogous to the KramersKronig relations, arguments of causality dictate that amplitude changes must be accompanied by phase changes. The dispersive loss therefore introduces a frequency-dependent loss and a frequency-dependent refractive index to the laser cavity. Consequently, there exist two mechanisms for correction of phase fluctuations. It will be shown that these mechanisms can be naturally modeled through modifications of the real and imaginary parts of the laser's complex susceptibility.

The dispersive loss technique can be easily implemented using different schemes of optical feedback, such as the reflection from an external cavity [6]. The external optical feedback serves as an effective loss (gain) mechanism in the semiconductor laser cavity. In an external cavity laser, one of the laser facets is used with an external mirror to compose a coupled-cavity system thereby rendering the facet's effective reflectivity (i.e., laser loss rate) frequency-dependent. When discussing the coupling of an external cavity to the laser cavity it is important to distinguish between internal and external losses. As will be discussed below, the external cavity can possess its 
own dispersive loss (external) but the dispersive loss the laser perceives (internal) will in general be different due to the coupling between the two cavities. In fact, in a simple external cavity laser the external loss only displays frequency-dependent phase changes while the internal loss is comprised of both frequency-dependent phase and amplitude changes. Both mechanisms have been shown to be instrumental in reducing the noise [4].

There is a tradeoff, however, in ordinary external cavity systems between linewidth reduction and effective bandwidth [8]. Relatively short cavities, having large effective bandwidths, can produce damping of relaxation oscillations but leave much to be desired in terms of linewidth reduction. To achieve substantial reduction one needs to employ long cavities and operate at relatively high feedback levels. Such conditions often lead to either mode hopping or multimode operation due to the closely spaced external cavity modes [9], [10]. In this paper, we will show that these problems can be circumvented by increasing the slope of the loss rate versus frequency to allow operation at lower feedback levels and to improve mode discrimination.

A frequently used technique is the formation of an external cavity with a high- $Q$ confocal Fabry-Perot (CFP) cavity acting as the reflector [8], [11]. This method performs quite well in terms of noise reduction and mode discrimination by "locking" the laser frequency to the CFP cavity resonance. However, since the dispersive loss is related to the resonant reflection from the CFP cavity, variations in the locking frequency can occur from unavoidable thermal and mechanical instabilities. We have recently demonstrated an alternative method using an atomic resonance, useful for both its spectrally narrow bandwidth (i.e., large slope) and inherent frequency stability. We have chosen to use the Faraday rotation in an atomic cesium vapor as the dispersive loss mechanism. Through the use of counter-propagating pump and probe beams a doppler-free reflection with a power broadened bandwidth of $\sim 20 \mathrm{MHz}$ is possible. Due to the small bandwidth of the reflection, small frequency variations in the laser field cause the dispersive loss to produce, in response, large changes in the field's amplitude and/or phase which are then fed back to the diode via the external cavity. With this technique, linewidth reductions as large as 2000 have been demonstrated at relatively small feedback levels [4].

In the particular geometry of our system, one should exercise care when discussing linewidth reduction. An important distinction must be made between reduction due to the atomic dispersive loss and reduction due to the storage of energy in the external cavity. It has been shown [7] that in an external cavity laser the linewidth reduction behaves as the ratio of stored energy in the cavity to the stored energy in the diode. The fact that in the atomic vapor arrangement the linewidth reduction is due to the atomic dispersive loss and not a consequence of the stored energy in the external cavity has been verified experimentally [4].
The purpose of this paper is to expand the theoretical framework for "locking" a semiconductor laser to the dispersive losses in an atomic vapor. The generic theory of noise reduction via dispersive losses is taken up in Section II through a van der Pol analysis. In Section III we compare the results of the van der Pol analysis to a detailed rate equation analysis specific to our system. Section IV gives the behavior of the lasing frequency and linewidth reduction under various conditions, as obtained through numerical simulations. The theory concentrates more on the semiconductor laser physics rather than on the effects within the atomic dispersive loss itself. The details of the atom-radiation field interactions will be presented elsewhere [12].

\section{VAN DER POL ANALYSIS}

The configuration of coupling the dispersive loss to the laser diode via an external cavity is shown in Fig. 1. In this three mirror configuration it is assumed that multiple reflections in the external cavity can be neglected since the feedback level is taken to be small. Within the external cavity is an atomic cesium cell placed between crossed polarizers which provides the dispersive loss [4], [13]. The cesium transition being used is the $852.1 \mathrm{~nm}$ cycling transition between the $F=4$ hyperfine level of $6 s_{1 / 2}$ and the $F^{\prime}=5$ hyperfine level of $6 p_{3 / 2}$ (see inset). The application of a weak axial magnetic field introduces a circular birefringence in the vapor by removing the degeneracy within the hyperfine levels. When the laser frequency is in spectral proximity of the cesium transition the plane of polarization is Faraday rotated in the cell and a fraction of the field is transmitted through the second polarizer. After reflection from the external mirror, the beam is again passed through the cell and returned to the laser diode. In this arrangement the counter-propagating pump and probe beams are orthogonally polarized with respect to each other (see Fig. 1). Through the use of a strong pump beam and weak crossed polarized probe beam it is possible to circumvent the Doppler broadening and obtain a spectrally narrow resonance [12].

The cesium dispersive loss introduces both a frequency-dependent loss and refractive index to the laser cavity. It will be shown in Section IV that the loss is symmetric in frequency about the cesium linecenter while the refractive index is antisymmetric. These properties are critical since the process of linewidth reduction relies on the slopes of these two curves.

To describe the effects of the dispersive loss we follow a van der Pol analysis in which the field intensity adiabatically follows the inversion density. For calculations of linewidth and chirp this treatment is justified [2]. The specific van der Pol analysis carried out below assumes that the dispersive loss is situated within the semiconductor laser cavity. Although our particular system configuration employs a loss mechanism external to the semiconductor, it will be shown that this calculation still provides the correct result for a loss introduced via an external optical feedback. 

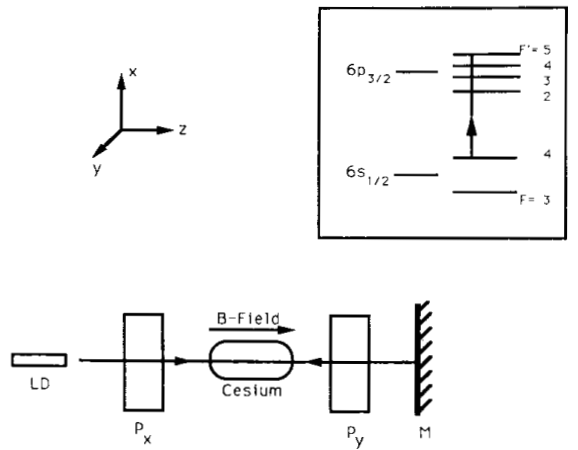

Fig. 1. Experimental arrangement for using Faraday rotation in atomic ce sium as a dispersive loss mechanism. LD: Laser Diode, M: External Mirror, P: Polarizer. Inset shows the relevant energy levels under consideration.

We begin by taking the optical field as

$$
E(t)=\left[A_{o}+\delta(t)\right] \exp \left\{i\left(\omega_{m} t+\varphi(t)\right)\right\}
$$

where $A_{o}$ is the field amplitude, $\delta$ and $\varphi$ are the smallsignal amplitude and phase $\left(\langle\dot{\varphi}\rangle_{t}=0\right.$, in which \langle\rangle$_{t}$ indicates temporal averaging), respectively, and $\omega_{m}$ is the lasing frequency. As shown in [2] the coupled rate equation for amplitude and phase is given by

$$
\begin{gathered}
2 i \omega_{m}\left(\dot{\delta}+i A_{o} \dot{\varphi}\right)+\frac{2 A_{o}^{2} \chi^{(3)}}{\mu^{2}}\left(2 i \omega_{m} \dot{\delta}-\omega_{m}^{2} \delta\right) \\
+\left(\omega_{n}^{2}-\omega_{m}^{2}+i \frac{\omega_{m}}{\tau_{p}}\right. \\
\left.-\left(\chi^{(1)}+A_{o}^{2} \chi^{(3)}\right) \frac{\omega_{m}^{2}}{\mu^{2}}\right) A_{o}=\Delta
\end{gathered}
$$

where the complex susceptibility $\chi$ is expressed as a nonlinear function of the lasing field by $\chi(E)=\chi^{(1)}+$ $\chi^{(3)}|E|^{2}$. The cold cavity resonant frequency is given by $\omega_{n}$, the nonresonant refractive index by $\mu$, and the photon lifetime by $\tau_{p} . \Delta$ is taken to be a Langevin noise source which will represent spontaneous emission. Following [3], the dispersive loss is modeled by a frequency-dependent loss rate as

$$
\frac{1}{\tau_{p}}=\frac{1}{\tau_{p o}}+2 C \dot{\varphi}
$$

where $C$ is a constant representing the slope of the loss curve, and the (instantaneous) lasing frequency is given by $\left(\omega_{m}+\dot{\varphi}\right)$. Since corrections to the loss rate are taken to occur instantaneously, the results of this analysis are valid only for frequencies smaller than the inverse of the longest response time of the system.

This ansatz describes changes in the field's amplitude due to the dispersive loss but not changes in the field's phase. To further take into account the effects of phase changes in the loss rate $C$ is defined as a complex number, $C=C_{r}+i C_{i}$. Using this form of $C$ in (3), we obtain the modified van der Pol equations for the amplitude and phase [4]

$$
\begin{gathered}
\dot{\delta}+\omega_{1} \delta+A_{o} C_{r} \dot{\varphi}=\Delta_{i} / 2 \omega_{m} \\
A_{o}\left(1+C_{i}\right) \dot{\varphi}-\alpha \omega_{1} \delta=-\Delta_{r} / 2 \omega_{m}
\end{gathered}
$$

where $\Delta_{r}$ and $\Delta_{i}$ are the real and imaginary parts, respectively, of the Langevin noise source, and

$$
\begin{aligned}
\omega_{1} & \equiv-\frac{\chi_{i}^{(3)} A_{o}^{2}}{\mu^{2}} \omega_{m} \\
\alpha & \equiv \frac{\chi_{r}^{(3)}}{\chi_{i}^{(3)}} .
\end{aligned}
$$

In (2) one can observe that the photon lifetime enters the rate equation on the same footing as the complex susceptibility. Therefore, the dispersive loss can be more naturally described through modifications in $\chi$, as opposed to a complex lifetime. Assuming the effects of the dispersive loss are well within the linear regime (i.e., ignoring the effects of saturation in the loss mechanism described through $\chi^{(3)}$, we can thus substitute

$$
\begin{gathered}
\chi_{r}^{(1)} \Rightarrow \chi_{r}^{(1)}+\frac{2 C_{i} \mu^{2}}{\omega_{m}} \dot{\varphi} \\
\chi_{i}^{(1)} \Rightarrow \chi_{i}^{(1)}-\frac{2 C_{r} \mu^{2}}{\omega_{m}} \dot{\varphi} .
\end{gathered}
$$

We see that frequency-dependent amplitude and phase changes are described by $C_{r}$ in $\chi_{i}^{(1)}$ and $C_{i}$ in $\chi_{r}^{(1)}$, respectively. Physically, $C_{r}$ represents a frequency-dependent loss and $C_{i}$ a frequency-dependent refractive index change in the semiconductor laser cavity.

To calculate the field spectrum linewidth we first calculate the autocorrelation function of the phase. This is readily obtained through Laplace transformation of $\delta$ and $\varphi[2]$ in (4) resulting in

$$
\begin{aligned}
\left\langle\varphi\left(t_{1}\right) \varphi\left(t_{2}\right)\right\rangle= & \frac{W}{4 A_{o}^{2} \omega_{m}^{2}} \frac{\left(1+\alpha^{2}\right)}{\left(1+\alpha C_{r}+C_{i}\right)^{2}} \\
& \cdot \min \left(t_{1}, t_{2}\right)
\end{aligned}
$$

where we have taken the correlations of the Langevin sources as

$$
\begin{aligned}
\left\langle\Delta_{i}\left(\lambda_{1}\right) \Delta_{i}\left(\lambda_{2}\right)\right\rangle & =\left\langle\Delta_{r}\left(\lambda_{1}\right) \Delta_{r}\left(\lambda_{2}\right)\right\rangle \\
& =W D\left(\lambda_{i}-\lambda_{2}\right) \\
\left\langle\Delta_{i}\left(\lambda_{1}\right) \Delta_{r}\left(\lambda_{2}\right)\right\rangle & =0 \\
W & =-\frac{4 h \omega_{m}^{3}\left(\frac{n_{2}}{\Delta n}\right)}{2 \pi \epsilon V \tau_{p o}}
\end{aligned}
$$

and $D(x)$ as the delta function. $V$ is the resonator volume, $\epsilon$ is the dielectric constant, and $\left(n_{2} / \Delta n\right)$ is the inversion factor. It should be noted that in this calculation of linewidth the noise source is taken to represent spontaneous 
emission (white noise) by choosing the noise correlation functions [(9)] to be delta-correlated in time.

The field autocorrelation function is now written as

$$
\begin{aligned}
& \left\langle E^{*}(t) E(t+\tau)\right\rangle \\
& \quad=A_{o}^{2} \exp \left[-\frac{\left\langle\varphi^{2}(\tau)\right\rangle}{2}\right] \times \exp \left(i \omega_{m} \tau\right)+\text { c.c. }
\end{aligned}
$$

After combining (8) and (12) and applying the WienerKhintchine theorem, we obtain a Lorentzian field spectrum with a spectral linewidth given by

$$
\Delta \nu=\Delta \nu_{\text {S.T. }} \frac{\left(1+\alpha^{2}\right)}{\left[1+\alpha C_{r}+C_{i}\right]^{2}} \equiv \Delta \nu_{\text {S.T. }} \frac{\left(1+\alpha^{2}\right)}{Q^{2}}
$$

where $\Delta \nu_{\mathrm{S} \text {.T. }}$ is the Schawlow-Townes linewidth [1] and $Q \equiv\left[1+\alpha C_{r}+C_{i}\right]$. It should be stressed that $C_{r}$ and $C_{i}$ describe the frequency-dependent amplitude and refractive index changes within the diode cavity. These will be referred to as the internal dispersive losses. The above treatment therefore applies to an arbitrary laser configuration, one of which is our scheme of external optical feedback.

As shown in (13), the field corrections made by the dispersive loss are twofold. An initial phase fluctuation causes a frequency shift in the laser field. On one hand, the dispersive loss will behave as a frequency discriminator and produce, in response, an amplitude change in the field described by $C_{r}$. Through the $\alpha$ parameter this amplitude change corrects for the initial phase fluctuation. On the other hand, the frequency shift in the laser field will cause the dispersive loss to produce, in response, a phase change in the field which will directly correct for the phase fluctuation. This process is described by $C_{i}$. Either separately or together, the two mechanisms reduce the rate of phase diffusion, thereby reducing the linewidth. Although frequency-dependent amplitude and refractive index changes both play a role in linewidth reduction, amplitude changes are weighted more heavily (for $\alpha>1$ ) since $C_{r}$ is multiplied by the $\alpha$ parameter.

\section{Static Operating Conditions}

To obtain expressions for $C_{r}$ and $C_{i}$ we need to investigate the operating conditions for our particular system. We now write the optical field inside the diode cavity $E(t)$ and its complex amplitude $\beta(t)$ as

$$
\begin{aligned}
& E(t)=\operatorname{Re}\left[\beta(t) e^{i \omega_{m t} t}\right] \\
& \beta(t)=\sqrt{P(t)} e^{i \varphi(t)}
\end{aligned}
$$

where $\langle P(t)\rangle_{t}$ is the average photon number in the mode. In the slowly varying amplitude approximation $\beta(t)$ can be described through the rate equation

$$
\dot{\beta}(t)=\frac{(1+i \alpha)}{2}(g-\gamma) \beta(t)
$$

where $g$ is the gain $\left(s^{-1}\right), \gamma$ is the loss rate, and $\alpha$ is the linewidth enhancement factor. To account for the coherent addition of an optical field from the external cavity to $\beta(t)$ we write (16) as [7]

$$
\begin{aligned}
\dot{\beta}(t)= & \frac{(1+i \alpha)}{2}(g-\gamma) \beta(t)-i\left(\omega_{m}-\Omega\right) \beta(t) \\
& +\kappa \beta(t-\tau) e^{-i \phi}
\end{aligned}
$$

where $\kappa$ is the feedback coupling rate defined by [9] $\kappa=$ $\pi r / F \tau_{d}, F$, and $\tau_{d}$ are the diode cavity finesse and roundtrip time, respectively, and $r$ is the external mirror reflectivity. The external cavity phase shift is given by $\phi=$ $\omega_{m} \tau, \tau=2 L / c$, being the round-trip time in the external cavity, and $\Omega$ is the lasing frequency without feedback.

The dispersive loss element in the external cavity is now modeled through a modification of $\kappa$ and $\tau$ as

$$
\begin{aligned}
& \kappa=\kappa(\omega)=\kappa\left(\omega_{m}\right)+\left.\frac{\partial \kappa}{\partial \omega}\right|_{\omega=\omega_{m}} \dot{\varphi} \\
& \tau=\tau(\omega)=\tau_{o}+\left.\frac{\partial \phi^{\prime}}{\partial \omega}\right|_{\omega=\omega_{m}}
\end{aligned}
$$

where $\phi^{\prime}$ is the phase shift due to the cesium $\left(\phi=\omega_{m} \tau_{o}\right.$ $\left.+\phi^{\prime}\right)$ and now $\tau_{o}=2 L / C$.

Substituting (18) into (17) and separating real and imaginary parts yields

$$
\begin{aligned}
\dot{P}(t)= & (g-\gamma) P(t)+2\left[\kappa\left(\omega_{m}\right)+\left.\frac{\partial \kappa}{\partial \omega}\right|_{\omega=\omega_{m}} \dot{\varphi}\right] \\
& \cdot \sqrt{P(t-\tau) P(t)} \cos \{\phi+\delta \varphi\} \\
\dot{\varphi}(t)= & \frac{\alpha}{2}(g-\gamma)-\left(\omega_{m}-\Omega\right)-\left[\kappa\left(\omega_{m}\right)\right. \\
& \left.+\left.\frac{\partial \kappa}{\partial \omega}\right|_{\omega=\omega_{m}} \dot{\varphi}\right] \sqrt{\frac{P(t-\tau)}{P(t)}} \sin \{\phi+\delta \varphi\}
\end{aligned}
$$

where $\delta \varphi=\varphi(t)-\varphi(t-\tau)$. In steady-state these equations give the operating conditions for the gain

$$
\Delta g=g-\gamma=-2 \kappa\left(\omega_{m}\right) \cos (\phi)
$$

and the lasing frequency

$$
\begin{aligned}
\Delta \omega & =\omega_{m}-\Omega \\
& =-\kappa\left(\omega_{m}\right) \sqrt{1+\alpha^{2}} \sin \left(\phi+\tan ^{-1}(\alpha)\right) .
\end{aligned}
$$

With the exception of the additional phase shift from the cesium, $\phi^{\prime}$ (contained in $\phi$ ), these equations are identical to those derived for an external cavity laser with no added losses [7].

Using the above results we can now obtain $C_{r}$ and $C_{i}$ for this system. Since the gain (loss) can be written as $g=\omega_{m} \chi_{i} / \mu^{2}$ [14], $C_{r}$ can be obtained from the slope of the loss curve [see (7b)] as

$$
\begin{aligned}
C_{r}= & \left.\frac{1}{2} \frac{\partial \gamma}{\partial \omega}\right|_{\omega=\omega_{m}}=\left.\frac{\partial \kappa}{\partial \omega}\right|_{\omega=\omega_{m}} \cdot \cos (\phi) \\
& -\kappa\left(\omega_{m}\right) \tau(\omega) \sin (\phi) .
\end{aligned}
$$


To derive $C_{i}$, the phase shift in the laser diode is written as $\Delta \varphi=\omega_{m} \tau_{c} \chi_{r} / 2 \mu^{2}$ [14]. This phase shift can also be obtained intuitively from the vector model of the complex amplitude [15] as $\Delta \varphi=\kappa \tau_{c} \sin (\phi)$. Therefore, we find from (7a) that

$$
\begin{aligned}
C_{i} & =\left.\frac{\partial[\kappa(\omega) \sin (\phi)]}{\partial \omega}\right|_{\omega=\omega_{m}} \\
& =\left.\frac{\partial \kappa}{\partial \omega}\right|_{\omega=\omega_{m}} \cdot \sin (\phi)+\kappa\left(\omega_{m}\right) \tau(\omega) \cos (\phi) .
\end{aligned}
$$

As discussed above, $C_{r}$ represents the frequency-dependent loss (internal) of the laser diode. It is seen in (23) that not only does the external loss (cesium vapor) contribute through $(\partial \kappa / \partial \omega)_{\omega=\omega_{m}} \cos (\phi)$ but the effect of coupling between the two cavities adds the term $\kappa\left(\omega_{m}\right) \tau(\omega)$ $\sin (\phi)$. Physically, this additional term represents the amplitude dependence on $\phi$ obtained from summing the internal and external (feedback) electric fields at the laser facet. Similarly, the frequency-dependent refractive index

$$
\begin{aligned}
& \frac{\partial \omega / \partial E}{\partial \Omega / \partial E}=\frac{\partial \omega}{\partial \Omega} \\
& =\frac{1}{\left(1+\alpha C_{r}+C_{i}\right)} \equiv \frac{1}{Q} \text {. } \\
& Q=1+\sqrt{1+\alpha^{2}}\left\{\kappa\left(\omega_{m}\right) \tau(\omega) \cos \left(\phi+\tan ^{1-1}(\alpha)\right)\right. \\
& \left.+\left.\frac{\partial \kappa}{\partial \omega}\right|_{\omega=\omega_{m}} \cdot \sin \left(\phi+\tan ^{-1}(\alpha)\right)\right\} .
\end{aligned}
$$$$
=\frac{1}{1+\sqrt{1+\alpha^{2}}\left\{\kappa\left(\omega_{m}\right) \tau(\omega) \cos \left(\phi+\tan ^{-1}(\alpha)\right)+\left.\frac{\partial \kappa}{\partial \omega}\right|_{\omega=\omega_{m}} \cdot \sin \left(\phi+\tan ^{-1}(\alpha)\right)\right\}}
$$

Numerical simulations (presented below) and recent experimental work [4] has shown that when a semiconductor laser is locked using this method, the laser tends to oscillate with minimum linewidth at a frequency corresponding to the peak of the cesium dispersive loss. Specifically, this behavior has been shown to occur when the laser frequency pulling is relatively small [see (22)] such that $\left(\phi+\tan ^{-1}(\alpha)\right) \approx 2 n \pi$. Under these conditions, $\left(\partial \phi^{\prime} / \partial \omega\right)_{\omega=\omega_{m}}$ plays more of a role than does $(\partial \kappa / \partial \omega)_{\omega=\omega_{m}}$. Therefore, the external frequency-dependent refractive index of the cavity [described through $\tau(\omega)$ ] is the important factor. However, the dominant mechanism for linewidth reduction is the internal frequency-dependent loss inside the semiconductor cavity described through $C_{r}$. This is seen by assuming an $\alpha$-pa-

Similarly, we can determine the frequency chirp due to changes in the cavity length as

$$
\frac{\partial \omega}{\partial L}=-\frac{4 \pi}{\lambda} \frac{\kappa\left(\omega_{m}\right) \cos \left(\phi+\tan ^{-1}(\alpha)\right)}{Q} .
$$

Therefore, the frequency locking stability of our system relative to an ordinary external cavity system is much better. As was verified experimentally, the reduction in frequency "jitter" can be more than an order of magnitude than that of a reasonably sized external cavity $(\sim 0.5 \mathrm{~m})$ [4].

\section{Rate Equation Analysis}

The result for linewidth reduction by a dispersive loss as predicted from the van der Pol model is applicable to any semiconductor laser system with optical feedback (under the constraints mentioned above). As previously stated, in the preceding analysis it was assumed that the loss mechanism was situated within the semiconductor laser cavity. The difference between an analysis of a loss mechanism within the semiconductor laser or external to it appears as a correction in the amplitude fluctuation terms in the rate equations. This arises from the effects of the finite delay time of the field in passing through the external loss mechanism. For calculating the spectral linewidth this does not affect the results, as will be shown. This rameter in the range of 4-5, yielding $\tan ^{-1}(\alpha) \approx \pi / 2$. Under the above conditions, $\phi \approx-\pi / 2$ so that $C_{r}[(23)]$ is much larger than $C_{i}[(24)]$. Moreover, as stated above, multiplication of $C_{r}$ by $\alpha$ further increases its effectiveness.

In Section IV an approximate model is presented for describing the cesium dispersive losses. A value of $Q \approx$ 700 is calculated. This represents a reduction of $2 \cdot 10^{4}$ below the Schawlow-Townes limit.

\section{Adiabatic Chirp Reduction}

Amplitude to phase coupling in semiconductor lasers manifests itself not only in enhancement of the spectral linewidth but also in FM generation under current modulation in a semiconductor laser. Frequency changes due to changes in injection current (chirp) are a direct consequence of the $\alpha$ parameter but can be reduced by the dispersive loss mechanism. Using (22) it is easily shown that the frequency chirp $(\Delta \omega)$ due to changes in injection current $(\Delta E)$ is reduced by 
shows the validity of the van der Pol method. It is independent of the particular configuration or geometry of the optical feedback. The key advantage of this analysis is that one only needs to calculate $Q$ from the system specific operating conditions. As a verification of this model we now calculate the linewidth reduction for our system by carrying out detailed dynamic calculations for the complex field amplitude and carrier density.

To calculate the laser linewidth we write the rate equation as

$$
\begin{gathered}
\dot{P}=(g-\gamma) P+\vartheta+2 P\left[\kappa\left(\omega_{m}\right)+\left.\frac{\partial \kappa}{\partial \omega}\right|_{\omega=\omega_{m}} \dot{\varphi}\right] \\
\cdot \sqrt{\frac{P(t-\tau)}{P(t)} \cos (\phi+\delta \varphi)+\Delta_{P}} \\
\dot{\varphi}=\frac{\alpha}{2}(g-\gamma)-\left(\omega_{m}-\Omega\right)-\left[\kappa\left(\omega_{m}\right)+\left.\frac{\partial \kappa}{\partial \omega}\right|_{\omega=\omega_{m}} \dot{\varphi}\right] \\
\quad \sqrt{\frac{P(t-\tau)}{P(t)}} \sin (\phi+\delta \varphi)+\Delta_{\varphi} \\
\dot{n}=-g P-\frac{n}{\tau_{s}}+E+\Xi
\end{gathered}
$$

where $\vartheta$ is the rate of spontaneous emission into the lasing mode, $\tau_{s}$ is the spontaneous lifetime, $E$ is the pumping rate, and $\Delta_{P}, \Delta_{\varphi}$, and $\Xi$ are Langevin noise sources. As before, these sources are taken to have zero mean and to be delta correlated in time (i.e., Markoffian [16]). The correlations and diffusion coefficients of the Langevin forces are given in (8) and (9).

The rate equations can be linearized about small variations using

$$
\begin{aligned}
& P(t)=P_{o}+\rho(t) \\
& n(t)=n_{o}+\eta(t) \\
& g(n)=g\left(n_{o}\right)+g^{\prime} \eta \quad\left(g^{\prime}=\frac{\partial g}{\partial n}\right) .
\end{aligned}
$$

The time delayed quantities in (9) can be Taylor expanded as in [7] to give

$$
\begin{aligned}
\sqrt{\frac{P(t-\tau)}{P(t)}} & \cong 1-\frac{\tau \dot{\rho}}{2 P_{o}} \\
\delta \varphi & =\varphi(t)-\varphi(t-\tau) \cong \tau \dot{\varphi} .
\end{aligned}
$$

Equation (14) relies on the assumption that the delay time $\tau$ is much smaller than the coherence time, which is valid for our cavity length and observed linewidths. This linearization leads to a system of coupled linear differential equations:

$$
\begin{gathered}
\dot{\rho}\left(1+a_{\phi}\right)+2 P_{o} \dot{\varphi}\left(b_{\phi}-a_{\kappa}\right) \\
=g^{\prime} P_{o} \eta-\frac{\vartheta}{P_{o}} \rho+\Delta_{P}
\end{gathered}
$$

$$
\begin{gathered}
\dot{\varphi}\left(1+a_{\phi}+b_{\kappa}\right)-\frac{b_{\phi}}{2 P_{o}} \dot{\rho}=\frac{\alpha}{2} g^{\prime} \eta+\Delta_{\varphi} \\
\dot{\eta}=-\left(\frac{1}{\tau_{s}}+g^{\prime} P_{o}\right) \eta-g\left(n_{o}\right) P_{o}+\Xi
\end{gathered}
$$

where $a_{\phi}$ and $b_{\phi}$ are the in-phase and quadrature components, respectively, of the frequency-dependent phase variations of the feedback field defined as [7]

$$
\begin{aligned}
& a_{\phi}=\kappa\left(\omega_{m}\right) \tau(\omega) \cos (\phi) \\
& b_{\phi}=\kappa\left(\omega_{m}\right) \tau(\omega) \sin (\phi)
\end{aligned}
$$

and $a_{\kappa}$ and $b_{\kappa}$ are the in-phase and quadrature components of the frequency-dependent amplitude variations of the feedback field defined as

$$
\begin{aligned}
& a_{\kappa}=\left.\frac{\partial \kappa}{\partial \omega}\right|_{\omega=\omega_{m}} \cos (\phi) \\
& b_{\kappa}=\left.\frac{\partial \kappa}{\partial \omega}\right|_{\omega=\omega_{m}} \sin (\phi) .
\end{aligned}
$$

This linear system of coupled differential equations (35) can be readily solved by taking the Fourier transform of the equations and then algebraically solving for the transformed functions $\hat{\rho}(\omega), \hat{\varphi}(\omega)$, and $\hat{\eta}(\omega)$. Instead of then carrying out the inverse transformations we use the method of Henry [17] to calculate the variance of the phase diffusion as

$$
\left\langle\Delta \phi(t)^{2}\right\rangle=\frac{1}{\pi} \operatorname{Real}\left[\int_{-\infty}^{\infty} d \omega\left\langle|\hat{\phi}(\omega)|^{2}\right\rangle\left(1-e^{i \omega t}\right)\right]
$$

where the Fourier transform of the phase is given by

$$
\begin{aligned}
\hat{\phi}(\omega)= & \frac{A_{P} \hat{\Delta}_{P}(\omega)+A_{\varphi} \hat{\Delta}_{\varphi}(\omega)+A_{n} \hat{\Xi}(\omega)}{\operatorname{Det}} \\
\operatorname{Det}= & i \omega\left\{g\left(n_{o}\right) g^{\prime} P_{o}\left[\alpha\left(b_{\phi}-a_{\kappa}\right)-\left(1+a_{\phi}+b_{\kappa}\right)\right]\right. \\
& -\left(i \omega+\frac{1}{\tau_{s}}+g^{\prime} P_{o}\right)\left[\left(i \omega\left(1+a_{\phi}\right)+\frac{\vartheta}{P_{o}}\right)\right. \\
& \left.\left.\cdot\left(1+a_{\phi}+b_{\kappa}\right)-i \omega b_{\phi}\left(b_{\phi}-a_{\kappa}\right)\right]\right\}
\end{aligned}
$$

The $A$ coefficients in (41) are the same as those given in [7] for an external cavity laser.

Since we are only interested in the linear time dependence of $\left\langle\Delta \varphi(t)^{2}\right\rangle$ (i.e., phase diffusion behaving as Brownian motion) in calculating the linewidth, (40) is evaluated by contour integration only considering the second-order pole at $\omega=0$ [17]. (The additional poles are related to the frequency and damping rate of relaxation oscillations.) After a substantial amount of algebra this integration leads to

$$
\left\langle\Delta \varphi(t)^{2}\right\rangle=\frac{\vartheta}{2 P_{o}} \frac{1+\alpha^{2}}{\left(1+\alpha C_{r}+C_{i}\right)^{2}} t=2 \pi(\Delta \nu) t
$$


where small quantities have been neglected as discussed in [17], and $C_{r}$ and $C_{i}$ are as defined in (23) and (24). Using the definition of the modified Schawlow-Townes linewidth [2], $\Delta \nu_{o}=\vartheta\left(1+\alpha^{2}\right) / 4 \pi P_{o}$, the expression for linewidth reduction becomes

$$
\frac{\Delta \nu}{\Delta \nu_{o}}=\frac{1}{\left[1+\alpha C_{r}+C_{i}\right]^{2}}=\frac{1}{Q^{2}} .
$$

Therefore, the linewidth reduction behaves as $1 / Q^{2}$, identically as predicted by the van der Pol analysis.

\section{Discussion}

In order to investigate the characteristics of a semiconductor laser "locked" to the dispersive loss, one can numerically solve for various quantities as a function of the lasing frequency using the above equations. The approach of our algorithm is shown in Fig. 2. Solutions to the frequency pulling equation [(22)] are shown graphically as the intersection of $(\omega-\Omega)$ and $B(\omega)$, the right-hand side of (22). The gain at this frequency is compared to the gain without feedback $(\Delta g=0)$ and if it is less, lasing occurs at this frequency. In Fig. 2 the gain condition is represented by the function $G(\omega)$. This function describes the difference between the required stimulated emission rate into the lasing mode with and without optical feedback [see (21), where $G(\omega)=\Delta g$ ] necessary to obtain lasing (i.e., zero net gain). As would be expected, near the cesium resonance $G(\omega)$ becomes negative indicating that less stimulated emission is required to lase compared to the case of no optical feedback.

As a simplistic first-order model of the cesium dispersive loss we assume a four-level system in the presence of a uniform magnetic field (applied along the propagation axis). Although the actual ground state $(F=4)$ and excited state $\left(F^{\prime}=5\right)$ possess nine-fold and eleven-fold degeneracies, respectively, we approximate both levels as being two-fold degenerate. In addition to the dispersive loss, other factors in the external cavity affect the loss rate. These "cavity losses" include losses from optical components as well as losses due to the inefficiency of coupling the feedback field into the lasing mode. Absorption in the atomic vapor is neglected in calculating the rotation but is taken into account in the cavity loss (including saturation effects)

In (17) the external cavity was characterized by a feedback coupling rate defined as $\kappa=\pi r / F \tau_{d}$ where $r$ is the external mirror reflectivity, and a phase shift $\phi$. With the Faraday rotation we assumed $\kappa$ to be frequency-dependent and added a phase shift $\phi^{\prime}$ (due to the cesium) to $\phi$. We now take $r$ and $\phi^{\prime}$ as

$$
\begin{aligned}
r & =r(\omega)=\frac{\omega L_{C s}}{c} \cdot\left(n_{+}-n_{-}\right) \\
\phi^{\prime}(\omega) & =\frac{\omega L_{C s}}{c} \cdot \frac{\left(n_{+}+n_{-}\right)}{2}
\end{aligned}
$$

where $L_{C s}$ is the length of the cesium cell, and $n_{+}$and $n_{-}$ are the refractive indexes of $\sigma_{+}$and $\sigma_{-}$circularly polar-

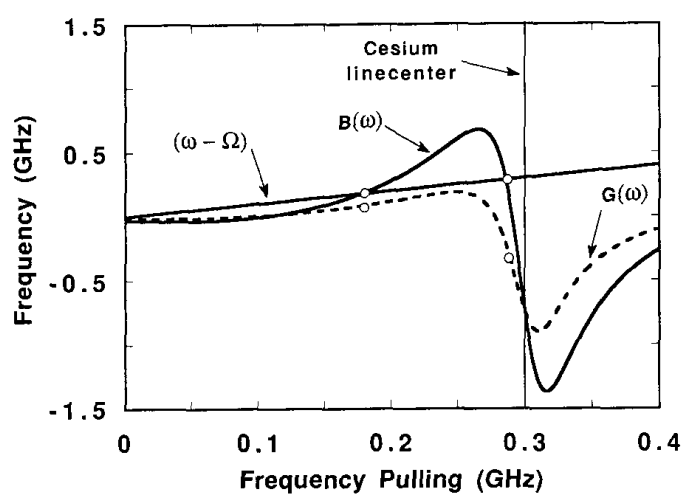

Fig. 2. Graphical interpretation of the algorithm used in modeling a semiconductor laser locked to a dispersive loss. $B(\omega)$ equals the right-hand side of (22) and $G(\omega)$ (dotted) equals the variation in gain given by (21). Offset frequency $\left(\omega_{o}-\Omega\right)=300 \mathrm{MHz}$, where $\omega_{o}$ is the dispersive loss linecenter frequency, and $\kappa_{\max }=1.0 \mathrm{GHz}$

ized light, respectively. This simple model ignores the basic physical mechanisms which give rise to the dopplerfree reflection. A full analysis of the complex multilevel cesium system as well as the coherent interaction of the crossed polarized pump and probe fields will be discussed elsewhere [12].

Fig. 3 shows the calculated quantities $\kappa(\omega)$ and $\phi^{\prime}(\omega)$ as a function of frequency. As previously stated, $\kappa$ is symmetric in frequency about the cesium linecenter while $\phi^{\prime}$ is antisymmetric. In the neighborhood of the linecenter, $\left(\partial \phi^{\prime} / \partial \omega\right)_{\omega=\omega_{m}}$ is positive and relatively constant while $(\partial \kappa(\omega) / \partial \omega)_{\omega=\omega_{m}}$ changes sign across the line. As discussed in [3], the sign of the slope of the loss curve determines whether the linewidth reduction or enhancement will occur. At first, one would expect reduction to be greater on the positive frequency side of $\kappa(\omega)$ (noting that $\kappa$ actually behaves as a frequency-dependent gain to the diode rather than a loss). However, (25) shows that not only is the sign of $(\partial \kappa(\omega) / \partial \omega)_{\omega=\omega_{m}}$ important but so is the sign of $\sin \left(\phi+\tan ^{-1} \alpha\right)$. Therefore, reduction can occur on either side of $k(\omega)$ depending on the sign of the frequency pulling as defined in (22).

We have considered the situation in which the lasing frequency is varied via a change in external cavity length. As shown in (28), a variation in the on-axis position of the external mirror will pull the lasing frequency away from $\Omega$. This behavior is used to scan the lasing frequency over the dispersive loss to observe the noise reduction. In addition to frequency pulling, we now define an offset frequency as the difference between the atomic cesium linecenter and the lasing frequency without feedback, i.e., $\left(\omega_{o}-\Omega\right)$. This quantity is shown to be important in determining the different roles of $C_{r}$ and $C_{i}$ in the noise reduction process.

In Fig. 4 the linewidth enhancement and the amplitude of the feedback field are plotted versus the lasing frequency. As shown in the figure, the linewidth reaches a minimum when lasing is at the top of the dispersive line. At this point the refractive index changes in the external 


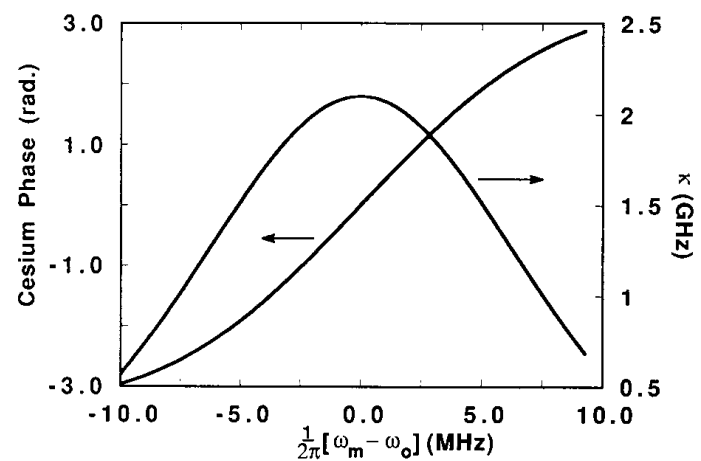

Fig. 3. Cesium dispersive loss quantities $\kappa(\omega)$ and $\phi^{\prime}(\omega)$ as calculated from the approximate model described in the text. Applied magnetic field $=1$ $\mathrm{G}$, temperature $=60^{\circ} \mathrm{C}$, power broadened transition linewidth $=30 \mathrm{MHz}$, Output intensity $=50 \mathrm{~mW} / \mathrm{cm}^{2}$, length of cesium $=5 \mathrm{~cm}$, saturation intensity $=1 \mathrm{~mW} / \mathrm{cm}^{2}$

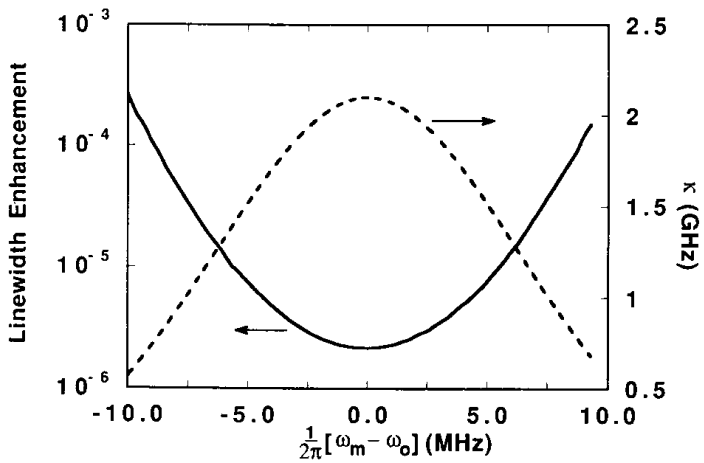

Fig. 4. Linewidth enhancement and feedback coupling rate $k(\omega)$ versus lasing frequency. Offset frequency $=0 \mathrm{MHz}, \kappa_{\max }=2.1 \mathrm{GHz}$.

loss are maximum while the amplitude changes are minimum. As discussed in [8] for the case of locking to a high- $Q$ CFP cavity, the phase corrections from the cesium can be viewed as increasing the effective time constant of the feedback system. Since the dispersive loss can be very narrow in our technique $(-17 \mathrm{MHz}$ bandwidth measured for the doppler-free reflection [4]), under our operating conditions $\left(\partial \phi^{\prime} / \partial \omega\right)_{\omega=\omega_{m}}$ can be much larger and in fact dominate $\tau_{o}$ in the expression for $\tau(\omega)$. In contrast to the ordinary external cavity laser, this system relies more on the frequency dependence of the optical feedback than on its magnitude. It can therefore produce large reduction at low feedback levels alleviating the problems of mode hopping.

Although the dominant effect in the reduction process is seen to be the frequency-dependent refractive index changes in the external loss, the amplitude changes described through $(\partial \kappa(\omega) / \partial \omega)_{\omega=\omega_{m}}$ can also play a role. In Fig. 5 the position of minimum linewidth relative to the cesium linecenter (detuning) is plotted as a function of offset frequency. At large offset frequencies minimum linewidth operation occurs slightly to the side of the dispersive loss linecenter indicating that amplitude changes are now contributing to the reduction.

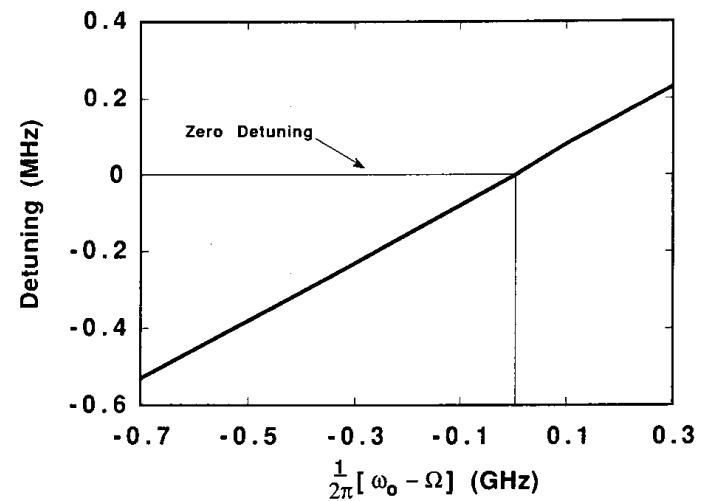

Fig. 5. Position of minimum linewidth relative to the cesium linecenter as a function of offset frequency. The position of zero detuning is marked on the graph. $\kappa_{\max }=2.1 \mathrm{GHz}$.

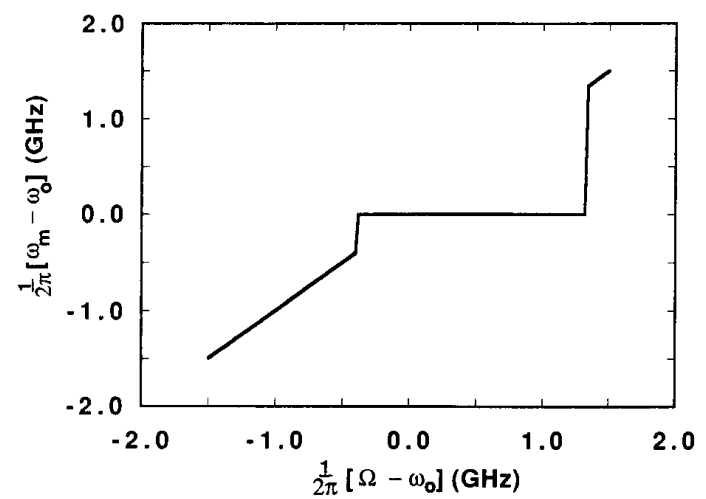

Fig. 6. Reduction in adiabatic chirp. Chirp reduction is given by the slope of the curve. Both frequencies are relative to the dispersive loss linecenter frequency $\omega_{0}$

In addition to linewidth reduction, frequency stability with respect to changes in injection current (chirp) is also improved using the dispersive loss. In Fig. 6 we show the actual lasing frequency $\omega_{m}$ plotted as a function of the lasing frequency with feedback $\Omega$. From the figure we see that if the lasing frequency is far from the cesium linecenter it follows the injection current as if there were no feedback present. However, in the neighborhood of the dispersive loss (in this case, within approximately 10-20 spectral bandwidths of the cesium resonance) the frequency "locks"' and the chirp is reduced by $1 / Q$. In addition, the chirp reduction is seen to be maximum near the top of the cesium line as would be expected from (27).

\section{SUMmary}

In conclusion, a van der Pol analysis has been carried out for a semiconductor laser with dispersive loss. To account for both amplitude and phase changes induced by the external loss mechanism, the internal laser loss rate is modeled through modifications to the complex susceptibility. It is shown that the linewidth reduction behaves as $1 / Q^{2}$ where $Q$ is a factor defined in terms of the geome- 
try-specific operating conditions of the semiconductor laser. In addition, adiabatic chirp is shown to decrease as $1 / Q$.

Although the analysis assumes the loss to be uniformly distributed throughout the laser, the results for linewidth reduction are also correct if the loss is introduced through a form of external feedback, as is the case in our method. This validity of the van der Pol analysis is shown by carrying out the calculation using the rate equations specific for our external loss.

Using an approximate model of the cesium dispersive loss, we calculate linewidth reduction and chirp reduction as a function of lasing frequency. The dominant mechanism of noise reduction was found to be the frequencydependent phase changes produced by the atomic vapor. However, under certain operating conditions, the vapor's frequency-dependent amplitude changes were also shown to play a role in the reduction process.

\section{ACKNOWLEDGMENT}

We thank M. Nakamura and N. Chinone of Hitachi's Central Research Laboratories for donation of the lasers used in the experimental investigations of this work.

\section{REFERENCES}

[1] A. L. Schawlow and C. H. Townes, "Infrared and optical lasers," Phys. Rev., vol. 112, pp. 1940-1949, 1958.

[2] K. Vahala and A. Yariv, "Semiclassical theory of noise in semicon ductor lasers-Part I," IEEE J. Quantum Electron., vol. QE-19, pp. 1096-1101, 1983.

[3] A. Yariv, R. Nabiev, and K. Vahala, "Self-quenching of the fundamental amplitude and phase noise with dispersive loss," Opt. Lett., vol. 15, pp. 1359-1361, 1990.

[4] Y. Shevy, J. Iannelli, J. Kitching, and A. Yariv, "Self-quenching of semiconductor laser linewidth below the Schawlow-Townes limit by using optical feedback," Opt. Lett., vol. 17, pp. 661-663, 1992.

[5] R. F. Kazarinov and C. H. Henry, "The relation on line narrowing and chirp reduction resulting from the coupling of a semiconducto laser to a passive resonator," IEEE J. Quantum Electron., vol. QE-23, pp. 1401-1409, 1987.

[6] K. Vahala and A. Yariv, "Detuned loading in coupled cavity semiconductor lasers-Effect on quantum noise and dynamics," Appl. Phys. Lett., vol. 45, pp. 501-503, 1984.

[7] G. P. Agrawal, "Line narrowing in a single-mode injection laser due to external optical feedback," IEEE J. Quantum Electron., vol QE-20, pp. 468-471, 1984.

[8] D. R. Hjelme, A. R. Mickelson, and R. G. Beausoleil, "Semiconductor laser stabilization by external optical feedback," IEEE $J$. Quantum Electron., vol. 27, pp. 352-372, 1991.

[9] R. Lang and K. Kobayashi, "External optical feedback effects on semiconductor injection laser properties," IEEE J. Quantum Electron., vol. QE-16, pp. 347-355, 1980.

[10] L. Goldberg, H. F. Taylor, A. Dandridge, J. F. Weller, and R. O Miles, "Spectral characteristics of semiconductor lasers with optical feedback," IEEE J. Quantum Electron., vol. QE-18, pp. 555-564, 1982.

[11] B. Dahmani, L. Hollberg, and R. Drullinger, "Frequency stabilization of semiconductor lasers by resonant optical feedback," Opt. Lett., vol. 12 , pp. 876-878, 1987.

[12] J. Iannelli, Y. Shevy, and A. Yariv, "Narrow spectroscopic resonances with saturation spectroscopy in atomic cesium," to be published.

[13] W. D. Lee and J. C. Campbell, "Optically stabilized AlGaAs/GaAs laser using magnetically induced birefringence in Rb vapor," Appl. Phys. Lett., vol. 58, pp. 995-997, 1991.

[14] A. Yariv, Quantum Electronics, 2nd ed. New York: Wiley, 1975, p. 318 .

[15] C. H. Henry, "Theory of the linewidth of semiconductor lasers," IEEE J. Quantum Electron., vol. QE-18, pp. 259-264, 1982.
[16] M. Lax, "Classical noise V. Noise in self-sustained oscillators," Phys. Rev., vol. 160, pp. 290-307, 1967.

[17] C. H. Henry, "Theory of the phase noise and power spectrum of a single-mode injection lasers," IEEE J. Quantum Electron., vol. QE-19, pp. 1391-1397, 1983

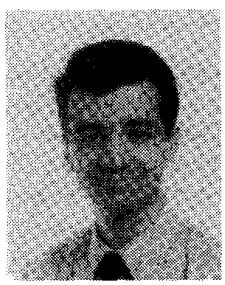

John M. Iannelli was born in Albany, NY, on June 4,1965 . He received the B.S, degree in physics, magna cum laude, from Rensselaer Polytechnic Institute in 1987 . He received the M.S. degree in applied physics from the California Institute of Technology, Pasadena, in 1990.

From 1987 to 1989 he was a member of the technical staff at the Jet Propulsion Laboratory, Pasadena, CA, where his research centered on the physics of III-V quantum-well structures and the infrared optical properties of metal particle composites. Currently, he is working towards the Ph.D. degree in applied physics also at the California Institute of Technology. His research interests include the effects of optical feedback on semiconductor lasers and the spectral dynamics of DFB and compound cavity lasers.

Mr. Iannelli is a member of the American Physical Society and the Optical Society of America.

Y. Shevy, photograph and biography not available at the time of publication.

J. Kitching, photograph and biography not available at the time of publication.

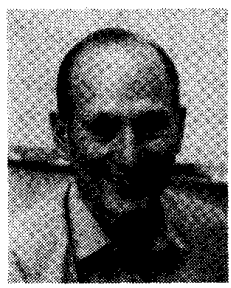

Amnon Yariv (S'56-M'59-F'70) was born in Is rael and served in the first artillery unit of the Israeli Defense Forces (1948-1950). He received the B.S., M.S., and Ph.D. degrees in electrical engineering from the University of California, Berkeley, in 1954, 1956, and 1958, respectively.

He joined Bell Telephone Laboratories, Murray Hill, NJ, in 1959 , at the early stages of the laser effort. He joined the California Institute of Technology, Pasadena, in 1964 as an Associate Professor of Electrical Engineering, and became Professor in 1966. In 1980 he was the Thomas G. Myers Professor of Electrical Engineering and Applied Physics. On the technical side, he took part (with various co-workers) in the discovery of a number of early solid-state laser systems, in proposing and demonstrating semiconductor based integrated optics technology, and in pioneering the field of phase conjugate optics. His present research efforts are in the areas of nonlinear optics, semiconductor lasers, and integrated optics. He has published widely in the laser and optics fields some 300 papers and has written a number of basic texts in quantum electronics, optics, and quantum mechanics. He is also a founder and chairman-of-the-board of the Ortel Corp., Alhambra, CA, and of Accuwave Corp.

Dr. Yariv is a member of the American Physical Society, Phi Beta Kappa, the American Academy of Arts and Sciences, and the National Academy of Engineering, and a Fellow of the Optical Society of America. He received the 1980 Quantum Electronics Award of the IEEE, the 1985 University of Pennsylvania Pender Award, the 1986 Optical Society of America Ives Medal, and the 1992 Harvey Prize. 